\title{
Reliability of the IOLMaster in axial length evaluation in silicone oil-filled eyes
}

\begin{abstract}
Purpose To assess the reliability of IOLMaster in axial length (AL) measurement in phakic silicone oil-filled $v$ s pseudophakic saline-filled eyes.

Methods Ten eyes of 10 patients, vitrectomized with silicone oil tamponade and scleral buckled with significant lens opacity were enrolled. Optical biometry with IOLMaster (Carl Zeiss Meditec AG, Germany) was performed 1 day before and 1 week after silicone oil removal and phacoemulsification with artificial intraocular lens (IOL) implantation in order to assess changes in $\mathrm{AL}$ measurements.

Results Mean AL was $26.16 \pm 1.23 \mathrm{~mm}$ (range $24.64-28.8 \mathrm{~mm}$ ) and $26.27 \pm 1.46 \mathrm{~mm}$ (range 25.26-29.6 mm), respectively, the day before and 1 week after silicone oil removal and cataract surgery, and the difference was not statistically significant $(P=0.2)$.

Conclusions Presence $v$ s absence of silicone oil tamponade as well as phakic $v s$ pseudophaphakic status in buckled and vitrectomized eyes did not influence the $A L$ measurement by means of no-contact optical biometry, suggesting that such eyes might be candidate for silicone oil removal and cataract surgery at one time.

Eye (2007) 21, 909-911; doi:10.1038/sj.eye.6702452; published online 28 July 2006
\end{abstract}

Keywords: axial length; laser interference biometry; vitrectomy; silicone oil; phacoemulsification; intraocular lens

\section{Introduction}

Intravitreal silicone oil injection is used to manage complicated retinal detachments (RDs). Corneal endothelial damage, band-shaped keratopathy, glaucoma, and cataracts are
M Parravano, F Oddone, M Sampalmieri and

D Gazzaniga well-known silicone oil-related side effects, ${ }^{1}$ which can be prevented or minimized with appropriate timing of silicone oil removal.

If cataract has occurred, silicone oil removal can be combined with cataract extraction and intraocular lens (IOL) implantation. ${ }^{2}$ The accuracy of IOL power calculation mainly relies on factors such as the accuracy of preoperative biometric data measurements, the IOL power calculation formulas, and the manufacturer IOL power quality control.

It has been previously reported that the reliability of axial length (AL) measurement performed with ultrasound biometers might be influenced by the presence of silicone oil in vitreous cavity, affecting the accuracy of IOL power calculation and thus postoperative refraction. ${ }^{3}$

The use of refraction data, AL measurement of the fellow eye, or AL ultrasound measurement before silicone oil injection ${ }^{4}$ was previously proposed as potential ways to avoid this bias. However, these methods might still lead to major IOL power measurement errors. ${ }^{5}$

In recent years, a noninvasive optical biometric method, based on partial coherence interferometry (PCI), was developed. Although acoustic biometry measures along the optical axis of the eye, the laser interference biometry (LIB) evaluates the length of the visual axis.

It has been demonstrated that the results obtained with PCI, using an internal, statistically verified, calculation algorithm, ${ }^{6}$ are comparable to ultrasonic immersion AL measurements in normal eyes.

The purpose of this study was to assess the reliability of IOLMaster AL evaluation in silicone oil-filled eyes.

Materials and methods

In this prospective longitudinal clinical study, 10 buckled, vitrectomized, and silicone oil-filled
Fondazione GB Bietti per lo Studio e la Ricerca in Oftalmologia-IRCCS Rome, Italy

Correspondence:

M Parravano,

Fondazione GB Bietti per lo Studio e la Ricerca in Oftalmologia-IRCCS,

Via Livenza 3,

Rome 00198, Italy

Tel: + 39 3498763147;

Fax: + 39620902968

E-mail: criparra@tin.it

Received: 2 January 2006 Accepted in revised form: 23 April 2006 Published online: 28 July 2006 
eyes of 10 patients were enrolled between May 2003 and June 2004. The reason for buckling and pars plana vitrectomy was a partial, not complicated RD, not involving the macula, in all patients. Inclusion criteria were age greater than 18 years, vitrectomy and silicone oil filling, partial not complicated RD with macula on, significant lens opacity, fully ability to understand and sign an informed consent.

Exclusion criteria were age-related maculopathy or other pathologies affecting the macula, corneal endothelial damage, band-shaped keratopathy, and glaucoma.

The patient's informed consent was obtained.

Ethical approval for the study was provided by the Ethical Committee at Fondazione GB Bietti per lo studio e la ricerca in Oftalmologia- IRCCS, Rome, Italy.

All patients underwent full vitrectomy with $1000 \mathrm{cSt}$ silicone oil injection (PDMS-Micromed, Rome, Italy) between 3 and 12 months before cataract surgery. An encircling silicone rubber band $(2.5 \times 0.7 \mathrm{~mm}$; Silibend, Micromed, Rome, Italy) was inserted behind the insertion of rectus muscles behind $14-15 \mathrm{~mm}$ the limbus in all eyes at the time of primary operation. All eyes presented significant lens opacity. Silicone oil removal was indicated in all of these eyes, as the retina was attached, combined with cataract extraction.

There was no contraindication in IOL insertion in any of these eyes.

Optical biometry was performed the day before and 1 week after silicone oil removal and cataract surgery.

Three consecutive AL measurements have been acquired by IOLMaster paying attention that the signal-to-noise ratio given by the machine was $>2.0$.

SRK/T formula was used to evaluate IOL power.

Surgery was performed by the same surgeon (MS), either under local or general anaesthesia. After conjunctival incision, two sclerostomies were performed (a lower temporal sclerostomy for the infusion cannula and an upper temporal sclerostomy for removal of the silicone oil) and then temporarily closed by plugs.

Phacoemulsification was performed through clear corneal incision and an acrylic foldable IOL (Alcon, Acrysoft MA60BM, $6 \mathrm{~mm}$ diameter) was implanted in the bag. The infusion cannula was then placed in the lower-temporal sclerostomy and silicone oil was aspirated actively.

Retinal evaluation was then performed and followed by partial fluid-air exchange.

\section{Results}

Ten eyes of 10 patients were recruited in this study (seven men, three women, mean age $63 \pm 6.82$, range $52-75$ years).
Mean AL values were $26.16 \pm 1.23 \mathrm{~mm}$ (range 24.6-28.8) and $26.27 \pm 1.46 \mathrm{~mm}$ (range 25.3-29.6) the day before and 1 week after the surgery, respectively. The difference between AL mean value the day before and 1 week after the surgery was not statistical significant $(P=0.2)$.

\section{Discussion}

The results of this study suggest that optical no-contact biometry may be used as a reliable method for AL measurements in patients with buckled, vitrectomized, and silicone oil-filled eyes, suggesting that such patients might be candidate for silicone oil removal and cataract surgery at one time.

It has been demonstrated from previous studies that the new LIB are reliable and safe for AL measurements both in aphakic and pseudophakic eyes. ${ }^{7,8}$

AL measurements with LIB are comparable with ultrasonic immersion measurements using an internal, statistically verified, calculation algorithm, ${ }^{6}$ and it has been demonstrated that this device is 10 times more accurate than the acoustic method in eyes with cataract and no other pathologies. ${ }^{9}$

Although acoustic biometry measures along the optical axis of the eye, the LIB evaluates the length of the visual axis and it is advisable if patients are able to fixate, especially in asymmetrically shaped eyes or patients with eccentric fixation. The emitted laser light has a wavelength of $780 \mathrm{~nm}$, which is close to the infrared spectrum and is visible to the patient. The AL is measured as optical path length between the anterior cornea and the retinal pigment epithelium that with a transformation equation is translated in geometrical distances. ${ }^{10}$ Moreover the no-contact procedure is more comfortable for the patient, reduces the risk of possible infection and also eliminates the risk of error caused by the pressure of the ultrasound probe on the corneal surface. $^{8}$

The calculation of IOL power in silicone oil-filled eyes have always represented a dilemma because conventional AL measurement methods can lead to gross mistakes. ${ }^{3}$ Ultrasonic AL measurement may give an artefactual longer AL-related higher refractive index of silicone oil (1.4035) compared with that of vitreous (1.33) that is responsible of a significant difference in sound velocity in these media. ${ }^{3}$

Different procedures have been proposed to solve the problem of AL measurement in these patients. Ghoraba et $a l^{11}$ tried to change the speed of ultrasounds, bringing it to $987 \mathrm{~m} / \mathrm{s}$, obtaining, however, unreliable values especially for extremely myopic eyes. Murray et $a l^{12}$ calculated a conversion factor of 0.71 to apply to the result obtained after ultrasonic biometry, usable only in eyes with silicone oil with a viscosity of $1300 \mathrm{cSt}$. Larkin 
et $a l^{13}$ used a factor of 0.64 multiplied by the measured vitreous cavity diameter with the silicone oil in the eye to obtain the actual vitreous cavity diameter. el Baha et $a l^{14}$ conducted intraoperative biometry after removing the silicone oil. However, all these methods demonstrated limits linked to either refraction defect and type of silicone used, or also because increase the risk of intraoperative infection.

It has also been proposed to measure $\mathrm{AL}$ preoperatively in all patients before silicone oil injection, and to use this measurement for IOL power calculation subsequently at the time of cataract extraction. This method is obviously limited when silicone oil tamponade is combined with scleral buckling, which may lead to major postoperative AL changes. ${ }^{4}$

Recently, the refractive outcome of silicone oil removal and IOL implantation using the IOLMaster have been studied. In that study, IOLMaster appeared to be a feasible and accurate method allowing reliable and accurate postoperative refractions, ${ }^{15}$ although no comparisons of AL measurements have been performed before and after silicone oil removal.

The aim of our study was to evaluate the reliability of AL measurement, which plays a major role in the ocular biometry and refraction correlating strongly with the subjective spherical equivalent, ${ }^{16}$ and not the refractive outcome that is the result of many variables.

In addition, within the first few weeks after cataract surgery in patients with 'clear cornea' surgery and implantation of foldable lenses, no significant postoperative change of the AL has been found. ${ }^{17}$

As expected in our study, mean AL values either 1 day before or 1 week after silicone oil removal and cataract surgery have been higher than values found in normal eyes, ${ }^{18}$ and this can be explained by the presence of scleral buckle that causes an elongation of the eyeball with a clear effect on the AL. ${ }^{19}$

In conclusion, the presence of silicone oil in vitreous chamber of patients with buckled and vitrectomized eyes does not influence AL measurement by means of nocontact optical biometry, suggesting that such patients might be candidate for silicone oil removal and cataract surgery at one time.

\section{References}

1 Federman JL, Schubert HD. Complications associated with the use of silicone oil in 150 eyes after retino-vitreus surgery. Ophthalmology 1988; 95: 870-876.
2 Baer RM, Aylward WG, Leaver PK. Cataract extraction following vitrectomy and silicone oil tamponade. Eye 1995; 105: 1234-1238.

3 Shugar JK, de Juan Jr E, McCuen II BW, Tiedeman J, Landers III MR, Machemer R. Ultrasonic examination of the silicone-filled eye: theoretical and practical considerations. Graefe's Arch Clin Exp Ophthalmol 1986; 224: 361-367.

4 Grinbaum A, Treister G, Moisseiev J. Predicted and actual refraction after intraocular lens implantation in eyes with silicone oil. J Cataract Refract Surg 1996; 22: 726-729.

5 Shioya M, Ogino N, Shinjo U. Changes in postoperative refractive error when vitrectomy is added to intraocular lens implantation. J Cataract Refract Surg 1997; 32: 1217-1220.

6 Rajan MS, Keilhorn I, Bell JA. Partial coherence laser interferometry vs conventional ultrasound biometry in intraocular lens power calculations. Eye 2002; 16: 552-556.

7 Findl O, Drexler W, Menapace R, Heinzl H, Hitzenberger CK, Ferch A. Improved prediction of intraocular lens power using partial coherence interferometry. J Cataract Refract Surg 2001; 27: 861-867.

8 Gantenbein C, Lang HM, Ruprecht KW, Georg T. First steps with the Zeiss IOLMaster: a comparison between acoustic contact biometry and non-contact optical biometry. Klin Monatsbl Augenheilkd 2003; 220: 309-314.

9 Drexler W, Findl O, Menapace R, Rainer G, Vass C, Hitzenberger $\mathrm{CK}$ et al. Partial coherence interferometry: a novel approach to biometry in cataract surgery. $A m \mathrm{~J}$ Ophthalmol 1998; 126: 524-534.

10 Hitzenberger CK. Optical measurement of the axial eye length by laser Doppler interferometry. Invest Ophthalmol Vis Sci 1991; 32: 616-624.

11 Ghoraba HH, El-Dorghamy AA, Atia AF, Ismail Yassin Ael-A. The problems of biometry in combined silicone oil removal and cataract extraction: a clinical trial. Retina 2002; 22: 589-596.

12 Murray DC, Durrani OM, Good P, Benson MT, Kirkby GR. Biometry of the silicone oil filled eye: II. Eye 2002; 16: 727-730.

13 Larkin GB, Flaxel CJ, Leaver PK. Phacoemulsification and silicone oil removal through a single corneal incision. Ophthalmologe 1998; 105: 2023-2027.

14 el-Baha SM, el-Samadoni A, Idris HF, Rashad KM. Intraoperative biometry for intraocular lens power calculation at silicone oil removal. Eur J Ophthalmol 2003; 13: 622-626.

15 Habibabadi HF, Hashemi H, Jalali KH, Amini A, Esfahani MR. Refractive outcome of silicone oil removal and intraocular lens implantation using laser interferometry. Retina 2005; 25: 162-166.

16 Touzeau O, Allouch C, Borderie V, Kopito R, Laroche L. Correlation between refraction and ocular biometry. $\mathrm{J} \mathrm{Fr}$ Ophtalmol 2003; 26: 355-363.

17 Falkenberg B, Kutschan A, Wiegand W. Analysis of optical parameters after cataract surgery and implantation of foldable lense. Ophthalmologe 2005; 102: 587-591.

18 Atchison DA, Jones CE, Schmid KL, Pritchard N, Pope JM, Strugnell WE et al. Eye shape in emmetropia and myopia. Invest Ophthalmol Vis Sci 2004; 45: 3380-3386.

19 Randleman JB, Hewitt SM, Stulting RD. Refractive changes after posterior segment surgery. Ophthalmol Clin N Am 2004; 17: 521-526, v-vi. 